\title{
A method for real-time condition monitoring of haul roads based on Bayesian parameter estimation
}

\author{
T Heyns ${ }^{\mathrm{a}, \mathrm{b}, *}$, PS Heyns ${ }^{\mathrm{c}}$, JP de Villiers ${ }^{\mathrm{a}, \mathrm{b}}$ \\ ${ }^{a}$ Department of Electronic and Computer Engineering, University of Pretoria \\ ${ }^{b}$ Defence Peace Safety and Security (DPSS) division of the Council for Scientific and Industrial Research (CSIR) of South Africa \\ ${ }^{c}$ Dynamic Systems Group, Department of Mechanical and Aeronautical Engineering, University of Pretoria
}

\begin{abstract}
Current haul road management techniques, such as routine, periodic and urgent maintenance have shortcomings in many complex haul road environments. Real-time road condition monitoring may significantly reduce maintenance costs, both to the road and to the vehicles. A recent idea is that vehicle on-board data collection systems could be used to monitor haul roads on a real-time basis by means of vibration signature analysis. This paper proposes a methodology based on Bayesian regression to isolate the effect of varying vehicle speed on the measured vehicle response metric. A key feature of the proposed methodology is that it avoids the costly need to generate analytical or empirical vehicle models.
\end{abstract}

Keywords: Monitoring of road condition, Bayesian parameter estimation, Condition based maintenance

\section{Introduction}

Haul roads are often unpaved and constructed from materials with diverse qualities. Not only are these roads subject to variable traffic volumes, but also to use by vehicle types which cover a range of different characteristics and payloads. Standard haul road maintenance management systems are generally poorly suited to deal with these complex and dynamic environments [1]. As a result many sub-optimal road maintenance strategies, such as routine, periodic and urgent maintenance [2], are implemented which generally may result in increased vehicle maintenance costs and reduction in service [1]. To combat these problems it has been proposed in the literature that on-board data collection systems could be used to perform real-time vibration monitoring of haul roads.

A number of model based road assessment methodologies have been proposed in the literature $[1,3,4]$. Thompson and Visser [1] propose that a Neural Network (NN) may be used to classify vibration patterns which are representative of different road conditions. Hugo et al. [3] investigate the use of an inverse vehicle dynamics model to estimate the road profile from a measured vehicle response, while Ngwangwa et al. [4] propose that the road profile may be estimated by means of a NN, rather than an analytic model. Hugo et al. and Ngwangwa et al. subsequently assess the estimated road profiles according to the ISO 8608 [5] road classification standards. Model based road classification techniques may potentially be very powerful, especially in environments where details of the road characteristics are required. Vehicle model estimation depends on either the characterisation of individual vehicle components [3], or the availability of manually prepared training data which should be representative of different

\footnotetext{
${ }^{*}$ Corresponding author

Email address: theoheyns@gmail.com (T Heyns)
} 
road conditions and vehicles speeds $[1,3]$. As such the vehicle modelling approach can be both challenging and expensive to implement in production orientated environments.

This paper assumes that many haul road environments do not necessitate detailed analysis of road characteristics. In many haul roads environments, such as opencast mines, the roads are of limited extent so that a site manager can inspect potentially problematic areas before maintenance decisions are made. Since these roads are usually constructed from gravel or asphalt it may be a comparatively low cost exercise to repair localized road defects, compared to the cost of replacing damaged tyres or repairing broken vehicles. For this kind of study it might suffice to represent the road condition by means of a simple vehicle response-type metric which is indicative of the adverse effect of the road on the vehicle.

The International Road Roughness Experiment [6] investigates a variety of possible metrics which can be used to quantify road roughness. They find that these vehicle response-type road roughness measuring systems (RTRRMS) are generally sensitive to both the road profile and the vehicle operating speed. For this reason the RTRRMS should be compared at a standardised speeds, or alternatively speed calibration functions should be used to account for the speed differences [6]. It is however also noted in the International Road Roughness Experiment that such calibration functions are dependent on the type and roughness of the surface which is traversed. This is because the RTRRMS reflects the presence of different wavebands and road signatures when operated over different surfaces at different speeds.

The methodology proposed in this paper exploits the fact that haul roads are generally traversed multiple times by either the same or similar vehicles. It is investigated how a speed-standardised RTRRMS may be estimated by means of regression functions which expresses the RTRRMS measurements as a function of speed. Individual regression functions are estimated for each of the road intervals, hence rendering it possible to reflect the unique spectral characteristics of the respective road intervals. More specifically this paper will investigate the use of Bayesian parameter estimation and regression to estimate a robust speed independent vehicle response metric based on the root mean square (RMS) of the measured acceleration.

\section{Methodology}

This paper proposes a low cost road monitoring methodology based on Bayesian parameter estimation and regression. In essence the methodology aims to fit unique regression functions to the vehicle response measurements which correspond to individual road intervals. By representing the road as a series (or grid) of individual road intervals it allows the interpolation functions to reflect the unique spectral characteristics of each specific road interval. The regression functions may subsequently be evaluated at a standardised speed to allow for more consistent comparison of the vehicle response.

This approach avoids the requirement for prior defined vehicle models or calibration functions. A RTRRMS is sensitive to the characteristics of the vehicle on which it is measured. Since haul roads are typically traversed by many different vehicle types, it is proposed to only instrument and consider the responses from a subset of similar (representative) vehicles.

One of the main challenges associated with fitting unique interpolation functions to each of the road intervals is the possible scarcity of data. This scarcity of data may be driven by three causes; namely that some roads may be less frequented, road intervals do not have uniform spectral characteristics and as such will result in data scatter (perceived as noise), and thirdly some sections may always be traversed at lower or higher speeds than the standardised speed so that the calibrated RTRRMS estimate will depend on extrapolation rather than interpolation. In an attempt to negate these problems use is made of Bayesian regression. In the Bayesian formalism both a data driven likelihood and a prior probability are considered during parameter estimation. This prior term allows experience from other sections of road to be fused with the observations made at a specific interval of road so as to obtain more robust regression functions. It is even possible to estimate an updated regression function for a road 
interval where only one observation has been made. The Bayesian approach also allows for an automated model selection criterion which selects the complexity of the implemented interpolation function based on the quantity and quality of observed data.

\subsection{Response-type road roughness measuring systems (RTRRMS)}

A large number of different RTRRMS metrics have been investigated in the literature. Some of the metrics are concerned with ride comfort (human response to vibration) such as the ISO 2631 standard [8]. This metric estimates the average power absorbed by the driver and is computed based on a frequency weighted acceleration RMS value as measured on the driver seat. Other metrics may be more concerned with the vehicle-road interaction forces, such as the metric proposed by Quinn and Wilson [7] which aims to represent the dynamic tire forces (and subsequently the road quality) based on the measured tyre pressures. The international road roughness index (IRI) was proposed as a standardised metric indication of road roughness based on a large scale study financed by the World Bank [6]. The IRI is based on the ratio of the accumulated suspension motion of a standard vehicle to the distance travelled by the vehicle during the measurement. The IRI may be estimated directly from a known road profile by simulating the response of a standardised quarter car vehicle at a constant speed of $80 \mathrm{~km} / \mathrm{h}$. The IRI has widely been adapted as a standardised measure of road roughness.

It is intended that the here proposed methodology should be relevant to most RTRRMS metrics. An experimental study is conducted where this idea is investigated on a single RTRRMS metric, namely the RMS acceleration as measured on the rear shaft axle of a utility vehicle (UV). The acceleration RMS is selected as a simple indication of the severity of the vibration (and thus the load) to which the vehicle is subjected.

As with other RTRRMS metrics the RMS response is not only sensitive to the road profile and the vehicle operating conditions, but also to the characteristics of the vehicle on which it is measured. For this reason it is advised that a single representative type of vehicle should be selected for any one haul road environment. The measurements obtained from these vehicles could then be compared in a consistent manner.

The merit of different RTRRMS metrics should be investigated further with regard to the demands of large vehicles and the additional maintenance criteria which are typical to haul road environments. It is therefore not the intention of this paper to necessarily promote the implemented RMS value. It anticipated that a study which investigates the correlation between a metric based on tyre pressures, or strain gauge measurements on specific vehicle components, and maintenance cost would be extremely interesting.

\subsection{Bayesian parameter estimation}

This section reviews the basics of Bayesian linear regression and indicates how it is implemented in the proposed methodology. The reader is referred to [11] for a comprehensive treatment on Bayesian parameter estimation.

Suppose that a grid has been defined which divides the road into various intervals. The vehicle response may be separated into segments associated with these different intervals based on a reference signal such as coordinates estimated by a global positioning system (GPS), reflective markers on the ground, or a combination of a push button and signal cross correlation alignment. The grid should be chosen such that the road condition over each section may be assumed to be fairly homogeneous.

A unique regression function (which explains the measured RTRRMS values as a function of speed) is fitted to each of the road intervals. These regression functions are estimated based on both the data driven likelihood and a parameter prior. The prior serves as indication of the typical nature of these interpolation functions. As such the prior allows for more robust interpolation functions, especially if only limited and noisy data are available.

Let the original RMS value which is associated with a specific road interval $j$ as measured at a mean speed of $v_{i}^{j}$ over that interval be denoted by $y_{i}^{j}$. Some variability in the vehicle response as measured over any road interval at 
a given speed is expected, since the road profile spectral characteristics are not perfectly uniform. This variability is accounted for by a noise term $n_{i}$, which is assumed to have a zero mean independent identically distributed Gaussian distribution $n \sim N\left(0, \sigma_{n}^{2}\right)$ with a constant variance $\sigma_{n}^{2}$.

An operating condition vector $\mathbf{x}$ is defined and implemented so that one vector $\mathbf{x}_{i}^{j}$ corresponds to each RMS datum point $y_{i}^{j}$. This operating condition vector determines the flexibility of the implemented regression function. The regression function in turn reflects the expected influence of operating conditions on the RTRRMS values. In this paper three simple regression functions are investigated, namely $\mathbf{x}=[1], \mathbf{x}=\left[\begin{array}{ll}1 & v\end{array}\right]^{\top}$ and $\mathbf{x}=\left[\begin{array}{lll}1 & v & v^{2}\end{array}\right]^{\top}$ with $v$ representing the vehicle speed. The three vectors respectively assume (i) speed independence, (ii) a linear dependence on speed, and (iii) a quadratic dependence on speed. The operating condition vector may easily be appended to also investigate higher order polynomial functions, or to also consider the influence of other variables such as vehicle acceleration. Bayesian model selection is used to select the most appropriate among the proposed interpolation functions.

An observation $y_{i}^{j}$ may now be described as the sum of the specific road interval interpolation function as evaluated for the corresponding operating condition vector $f\left(\mathbf{x}_{i}^{j}\right)$ and the noise term $n_{i}$.

$$
y_{i}^{j}=f\left(\mathbf{x}_{i}^{j}\right)+n_{i}
$$

If the operating condition vector is sufficiently expressive it may be justified to assume that the interpolation function can be approximated as having a linear dependence on $\mathbf{x}$. This linear dependence is expressed by the parameter vector $\mathbf{w}^{j}$ :

$$
f\left(\mathbf{x}^{j}\right) \approx\left\{\mathbf{x}^{j}\right\}^{\top} \mathbf{w}^{j}
$$

Any discrepancies in this assumption is absorbed by the noise term.

Let all the RMS measurements which correspond to a specific road interval be denoted by the vector $\mathbf{y}^{j}$, and let all the associated operating condition vectors be contained in the matrix $\mathbf{X}^{j}$. The likelihood of the data given the model as represented by the parameter values $\mathbf{w}^{j}$ may now be denoted $p\left(\mathbf{y}^{j} \mid \mathbf{X}^{j}, \mathbf{w}^{j}\right)$. Due to the independent noise assumption the joint likelihood for the $K$ RMS observations which corresponds to any road interval, may simply be computed as the product of the individual datum point likelihoods:

$$
\begin{aligned}
p\left(\mathbf{y}^{j} \mid \mathbf{X}^{j}, \mathbf{w}^{j}\right) & =\prod_{i=1}^{K} p\left(y_{i}^{j} \mid \mathbf{x}_{i}^{j}, \mathbf{w}^{j}\right) \\
& =N\left(\left\{X^{j}\right\}^{\top} \mathbf{w}^{j}, \sigma_{n}^{2} \mathbf{I}\right)
\end{aligned}
$$

The parameter estimates which optimize equation 4 is equivalent to the least square error (LSE) solution . It is assumed that there are a number of road intervals where sufficiently many measurements are available so that the LSE solution will be fairly good. Such intervals may automatically be selected based on certain criteria. The parameter values for those LSE estimates (as estimated at road intervals with good data) are used as guideline to what the typical parameter values are to be at other road intervals where sufficient data may not be available. A multivariate Gaussian distribution is estimated from the LSE solutions for the reference road intervals. This distribution is subsequently used as the prior distribution $p(\mathbf{w})$. Let the prior mean be denoted by the vector $\mathbf{m}_{0}$, and let the covariance matrix be denoted as $\mathbf{S}_{0}$, so that the prior may be expressed as:

$$
p(\mathbf{w})=N\left(\mathbf{w} \mid \mathbf{m}_{0}, \mathbf{S}_{0}\right)
$$


By Bayes' theorem the prior and the data driven likelihood may now be used to obtain a posterior distribution over the parameter values:

$$
\begin{aligned}
\text { posterior } & =\frac{\text { likelihood } \times \text { prior }}{\text { marginal likelihood }}, \\
p\left(\mathbf{w}^{j} \mid \mathbf{y}^{j}, \mathbf{X}^{j}\right) & =\frac{p\left(\mathbf{y}^{j} \mid \mathbf{X}^{j}, \mathbf{w}^{j}\right) p\left(\mathbf{w}^{\mathbf{j}}\right)}{p\left(\mathbf{y}^{j} \mid \mathbf{X}^{j}\right)},
\end{aligned}
$$

where the marginal likelihood $p(\mathbf{y} \mid \mathbf{X})$ serves to normalize the posterior. It may be shown that the posterior distribution is also a Gaussian distribution [11]:

$$
p\left(\mathbf{w}^{j} \mid \mathbf{y}^{j}, X^{j}\right)=N\left(\mathbf{w}^{j} \mid \mathbf{m}_{n}^{j}, \mathbf{S}_{n}^{j}\right)
$$

where the posterior mean $\mathbf{m}_{n}$ and covariance $\mathbf{S}_{n}$ for road section $j$ are given by:

$$
\begin{aligned}
\mathbf{m}_{n}^{j} & =\mathbf{S}_{n}\left(\mathbf{S}_{0}^{-1} \mathbf{m}_{0}+\sigma_{n}^{-2}\left\{\mathbf{X}^{j}\right\}^{\top} \mathbf{X}^{j}\right) \\
\mathbf{S}_{n}^{j} & =\left(\mathbf{S}_{0}^{-1}+\sigma_{n}^{-2}\left\{\mathbf{X}^{j}\right\}^{\top} \mathbf{X}^{j}\right)^{-1}
\end{aligned}
$$

The likelihood of observing a RMS value $y_{*}$ at an operating condition $\mathbf{x}_{*}^{j}$ while traversing road section $j$ may be estimated from the updated likelihood function which is again of Gaussian form [11]:

$$
p\left(y_{*} \mid \mathbf{y}^{j}, \mathbf{X}^{j}, \mathbf{m}_{0}, \mathbf{S}_{0}, \sigma_{n}\right)=N\left(y_{*} \mid \mathbf{w}_{n}^{\top} \mathbf{x}_{*}^{j},\left\{\tau_{*}^{j}\right\}^{2}\right)
$$

The variance $\left\{\tau_{*}^{j}\right\}^{2}$ of the predictive distribution indicates the uncertainty in a prediction at operating condition $\mathbf{x}_{*}^{j}$ and is given by:

$$
\left\{\tau_{*}^{j}\right\}^{2}=\sigma_{n}^{2}+\left\{\mathbf{x}_{*}^{j}\right\}^{\top} \mathbf{S}_{n} \mathbf{x}_{*}^{j}
$$

\section{Experimental setup}

\subsection{Measurements}

An accelerometer was attached to the rear wheel shaft. The vehicle response was measured with a DC coupled Crossbow accelerometer and recorded on a Somat eDAC-lite data acquisitioning system at a sampling frequency of $400 \mathrm{~Hz}$. The integrity of the DC coupled Crossbow accelerometer was tested on a high frequency actuator and found to be very accurate over the frequency range of interest. An infrared eye and a push button were also connected to the eDAC to provide reference signals. A proximity sensor on the drive shaft was used to measure its rotational speed. 
The vehicle speed was calculated from the measured drive shaft speed and from knowledge of the shaft-wheel rotation ratio and the effective rolling radius of the wheel. The vehicle's speed was integrated over time to obtain an estimated relative position vector. The acceleration signal was subsequently resampled to be a function of position rather than time.

Figure 1 depicts in (a) the measured raw vibration signal and in (b) the corresponding RMS severity metric. The severity metric is computed as the RMS acceleration over 1 metre intervals. The interval windows are selected so as to overlap the previous interval by a half metre. The RMS severity metric thus has a frequency of two datum points per metre.
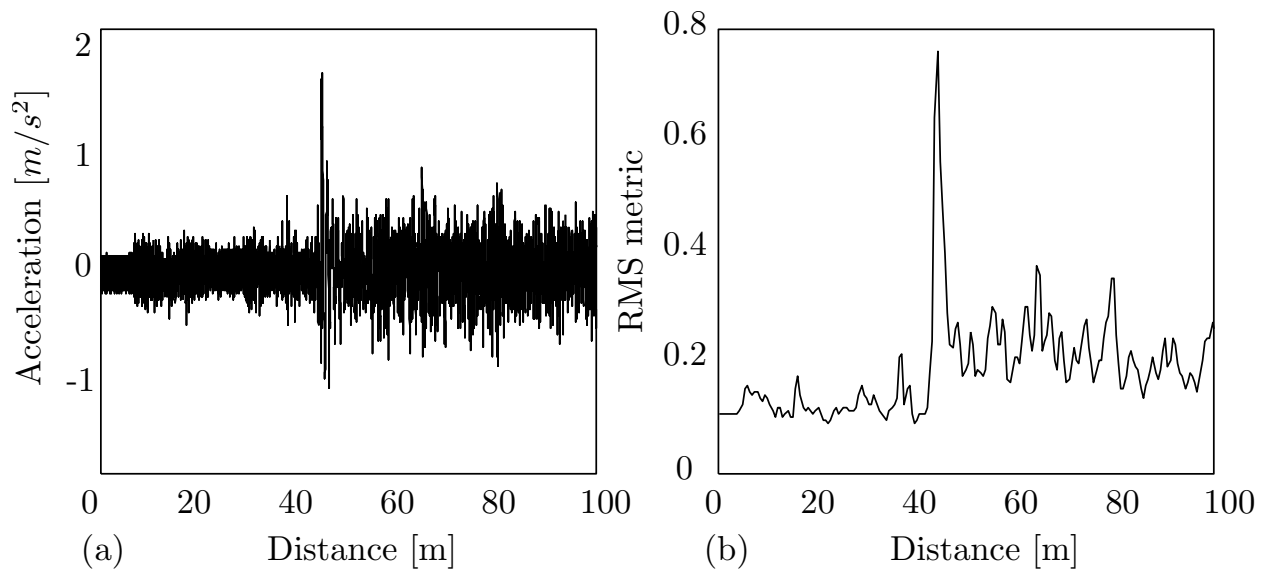

Figure 1: The severity metric is derived from the acceleration signal by computing its RMS over a 1 metre sliding window with 0.5 metre overlap

\subsection{Road profiles and signal alignment}

The response of the UV was measured both above and below ground. Two above ground road profiles with favourable characteristics were available on site. The UV response over these profiles were measured with the intention investigating the interpolation procedure over simple road profiles. Figure 2 (a) indicates how an impulse excitation was created by laying down a row of concrete blocks on a level surface. Figure 2 (b) illustrates the second profile which comprised two closely situated humps of similar dimension. The two humps were situated in a low wavelength ditch.
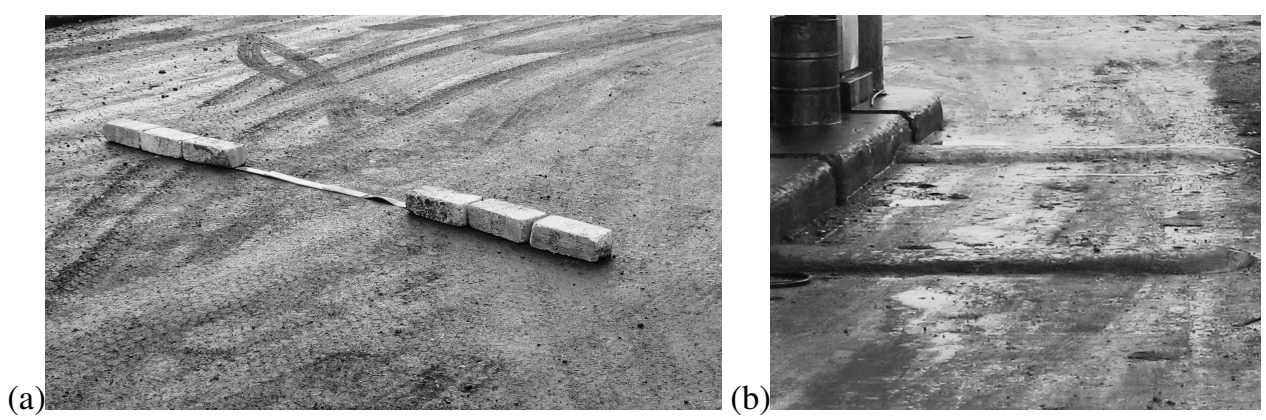

Figure 2: The known road profiles (a) impulse like excitation (b) double humps situated in ditch

The above ground road profiles were recorded by measuring the vertical distance between the road surface and a reference cable which was tensioned above the road. The vehicle's position relative to the road profiles was 
estimated by means of an infrared eye which detected reflective tape strips on the ground which was situated at the start and end of the runs.

Measurements were also performed underground. The UV response was measured over two $730 \mathrm{~m}$ long sections of underground road, which were identified as being representative of typical underground conditions. The UV was driven thrice over each of the two road sections. Due to the underground working conditions it was not possible either to measure the road surfaces, nor to use reflective tape to indicate the start and ends of each run. For this reason the vehicle responses were aligned by optimizing their cross-correlations.

\subsection{Parameter prior}

It has been explained that the Bayesian prior is used to improve the estimated parameter values for the regression functions and is especially important for road intervals which are subject to constrained data. The prior is based on general parameter values (and covariance's) as observed at other road intervals.

In this case study the measurements from one of the underground profiles is used to construct the prior. The road profile is $730 \mathrm{~m}$ long and was traversed three times. The RMS severity metric was computed with $0.5 \mathrm{~m}$ overlap so that there are a total of 1460 intervals. Using the standard least square error (LSE) criterion an interpolation function is estimated for each of the 1 metre intervals. The standard LSE solution is efficiently solved using the closed form analytic solution [11].

None of the road intervals have been traversed especially many times, so that a subset of representative road segments (where the parameter values are expected to be more accurate) will not be identified. Rather all the 1460 estimates (as based on the LSE criterion) will be used to estimate the prior over the parameter values. While it is expected that some of the LSE estimates may be very crude, it is still anticipated that the estimated distribution over all of the LSE estimates will tend toward a good representation of the true parameter ranges as associated with road intervals of different spectral characteristics.

The functional relationship between the vehicle speed and response is not known a priori. The process of computing the LSE solution for each road interval is therefore repeated for different modelling assumptions. In this paper three models are investigated which respectively assume that the vehicle response as measured over a specific section of road is either: (i) independent of speed, (ii) linearly dependent on speed or (iii) a quadratic function of speed. An individual prior over the parameter values is estimated for each of the possible modelling assumptions.

Each of the priors are approximated as a multivariate Gaussian density. This multivariate density indicates the expected parameter values, their variances, along with the covariance which exist between the parameter values. The prior is thus fully characterized by its mean $\mathbf{m}_{0}$ and covariance $\mathbf{S}_{0}$.

\subsection{Bayesian model selection}

Once the prior density distribution for each of the interpolation functions has been estimated, it remains to be determined which of the interpolation assumptions are most appropriate for the given data set. It is also required to estimate the observation noise variance $\sigma_{n}^{2}$. If the observation noise is assumed very small relative to the variance of the parameter values as expressed by the prior, then each data observation will be much trusted and thus significantly influence the posterior. Conversely, if the noise variance is assumed very large then many observations will be required to significantly influence the posterior parameter estimate.

The problem of both selecting the most appropriate interpolation function as well as the optimal noise variance may simultaneously be solved by means of Bayesian model selection. Let each of the three interpolation functions be paired with a range of values for the noise variance. Each pair represents a potential model. The most appropriate model is the one that optimizes the posterior probability as denoted in equation 6 . The posterior probabilities are based on all the data as measured over the first underground road profile of $730 \mathrm{~m}$ in length. 
The data driven likelihoods, the model priors, and the subsequent posterior probabilities for each of the possible models are computed. The results for the second and third order interpolation functions as paired with different values for the noise standard deviation are presented as graphs. For sake of brevity the results for the speed independent assumption is not presented, all evidence however clearly points to a speed dependence in the data.

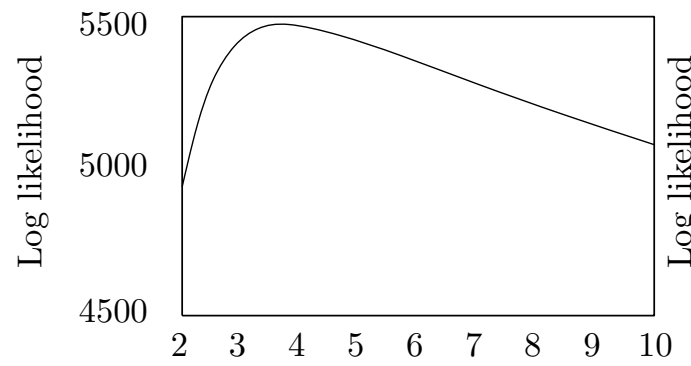

(a)

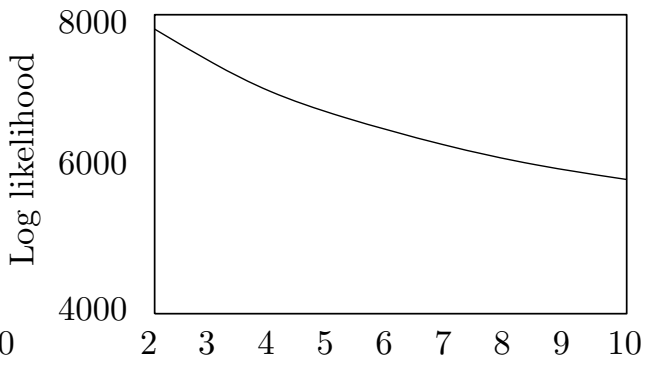

(b)

Figure 3: Logarithm of the likelihood term with respect to different noise standard deviations $\left[\sigma_{n}\right]$ as computed for (a) the first order and (b) the second order functions of speed

Figure 3 depicts the log likelihoods as computed over all the observations for all the road intervals for (a) the linear dependence on speed and (b) the quadratic dependence on speed. The log likelihood for the linear model reaches a maximum at a noise standard deviation of approximately $\sigma_{n}=3.5 \times 10^{-3}$. The log likelihood for the quadratic term outperforms that of the linear model, it never reaches a maximum but rather tends towards infinity as the noise standard deviation approaches zero. This reflects the fact that the quadratic interpolation functions are sufficiently flexible to perfectly model the three observations per interval.

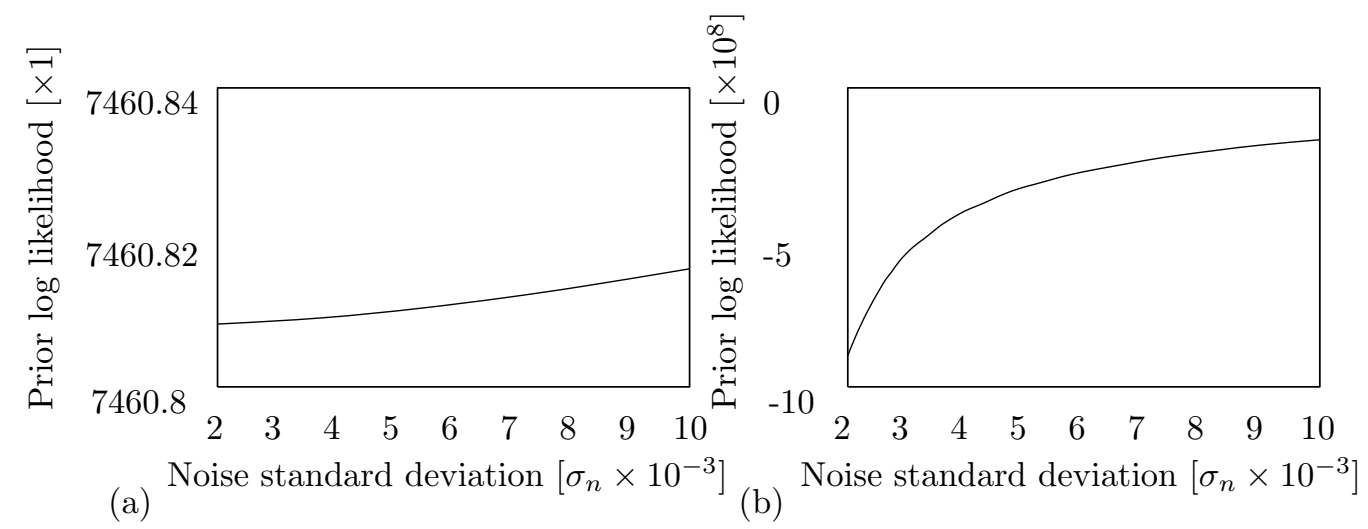

Figure 4: Logarithm of the prior probabilities with respect to different noise standard deviations $\left[\sigma_{n}\right]$ as computed for (a) the first order and (b) the second order functions of speed

The prior probabilities of the model parameters are computed and illustrated in figure 4 for (a) the linear dependence on speed and (b) the quadratic dependence on speed. A larger noise standard deviation resulted in parameter estimates which better corresponded to the prior means, hence resulting in a greater prior probability. Consider that the quadratic dependence requires the estimation of an additional 1460 parameter values. This additional flexibility significantly penalized the quadratic term, especially when the observation noise is assumed small. The simpler linear dependence (which spread its density distribution over fewer parameter) resulted in significantly higher parameter prior probabilities. 


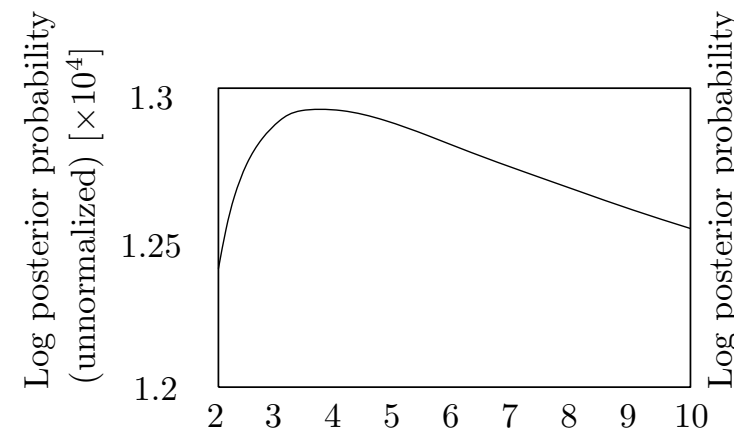

(a)

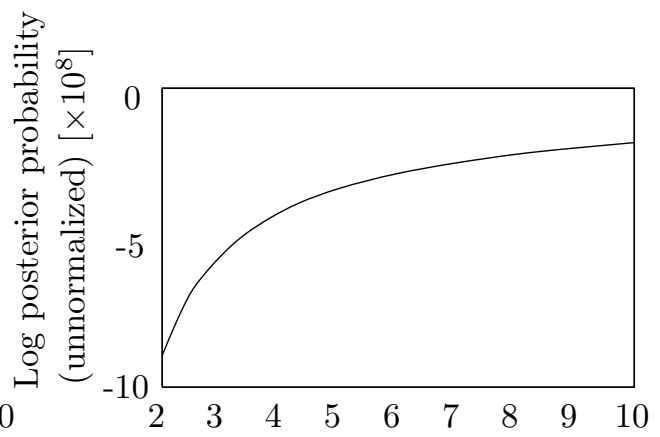

(b)

Figure 5: The logarithm of the unnormalized posterior probabilities with respect to different noise standard deviations $\left[\sigma_{n}\right]$ as computed for (a) the first order and (b) the second order functions of speed

A log posterior model probability is computed as the sum of the associated log likelihood, the log prior probability, and the negative log marginal probability. The marginal probability expresses the probability of observing the data given all of the assumed possible models. The marginal probability reduces to a constant value which acts as a normalizing term to all of the posteriors. Figure 5 ignores the marginal term and subsequently compares the unnormalized posterior probabilities for both (a) the linearly dependencies with different noise standard deviations and (b) the quadratic dependencies as computed with different noise standard deviations. The model with the maximum posterior probability corresponds to a linear dependence on speed, with a noise standard deviation of approximately $3.7 \times 10^{-3}$. If the posteriors are normalized with respect to the different speed dependencies (independent, linear or quadratic) then the posterior of the linear assumption is virtually 1 , with the other two model posteriors effectively equalling 0 .

The linear dependence on speed assumption clearly proves to the most appropriate this data set. It is important to note that these results do not prove the optimality of a linear assumption for other RMS data sets. It is very possible that higher model orders would be favoured if more data per interval are available.

The results presented in figures 3, 4 and 5 are based on the assumption that a single type of regression function should be fitted to all of the road intervals. This is by no means a requirement. The most appropriate type of regression function for any individual road interval may automatically be updated as more measurements become available and the posterior begins to favour more accurate interpolation functions.

\subsection{Iterative updating}

The Bayesian approach allows for the iterative updating of the posterior function with each data observation. After an observation for a section has been made and the posterior has been computed the posterior will be used as the prior for the next observation [11]. The solution is mathematically equivalent to the solution obtained by considering all the data simultaneously, however it avoids the requirement of storing any data. 


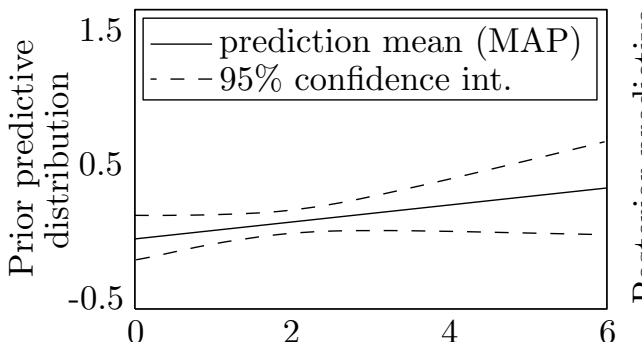

(a)

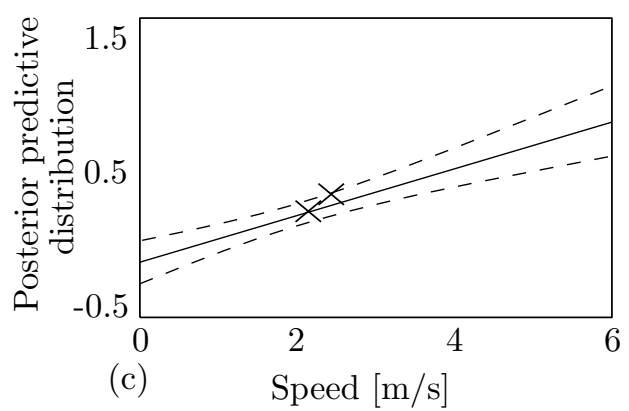

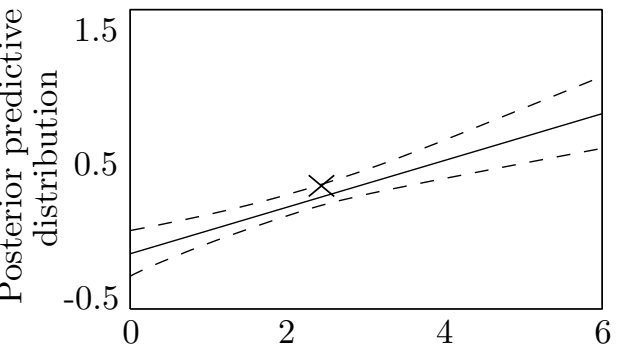

(b) Speed $[\mathrm{m} / \mathrm{s}]$

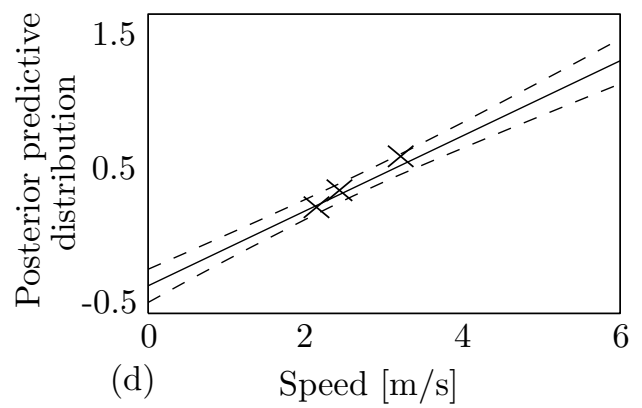

Figure 6: Updating of the estimated regression function as more measurements become available, starting from (a) the prior, (b) one observation, (c) two observations, (d) three observations

Figure 6 illustrates the growing confidence in the interpolation function as more measurements become available. The prior function (linear assumption) is illustrated in figure 6 (a). Two dashed lines are used to indicate the two standard deviation bounds (95\% confidence interval). The prior confidence bounds are fairly weak, thus indicating that the regression function is still uncertain. Figure 6 (b), (c) and (d) depicts how the intercept and slope adapt as more measurements become available. The confidence over the interpolated values increases accordingly.

\section{Results}

The priors and the observation noise variance have been estimated from data which are representative of typical underground road conditions as measured by a specific UV. The ability of the proposed regression function approach to estimate speed standardised RMS values is subsequently investigated on the two above ground roads of which the profiles have been measured. The UV was driven over the concrete blocks at four different speeds, resulting in four measurements; $m_{1}$ to $m_{4}$. Figure 7 (a) depicts a level road with a sudden impulse like excitation due to the concrete blocks. The vehicle response is underdamped so that the energy takes some time to dissipate from the system. All of the measurements, including the underground runs, were conducted at speeds which vary between 1 and $4 \mathrm{~m} / \mathrm{s}$. These slow speeds were either mandated by the poor road conditions, or safety precautions. A standardised speed of $3 \mathrm{~m} / \mathrm{s}$ was selected as the speed which was most adept to interpolation. From figures 7 (b) and (c) it may be seen that the RMS severity significantly depends on the vehicle speed. The $3 \mathrm{~m} / \mathrm{s}$ standardised severity metric is presented in figure 7 (d). The solid line $\left(m_{1}\right)$ indicates the estimated RMS value at $3 \mathrm{~m} / \mathrm{s}$ (also referred to as the maximum a posteriori (MAP) solution), while the two standard deviations dashed lines indicate the $95 \%$ confidence bounds. It is seen that the RMS values which represents the vehicle passing over the concrete blocks as measured at higher speeds (more than $3 \mathrm{~m} / \mathrm{s}$ ) are almost double the magnitude of the measurement made at a low speed (about $1.5 \mathrm{~m} / \mathrm{s}$ ). Those RMS values which were measured over the intervals of smooth road were near speed independent. Figure 7 (d) indicates that the localized regression functions were able to account for the different dependencies on speed for different road intervals. 

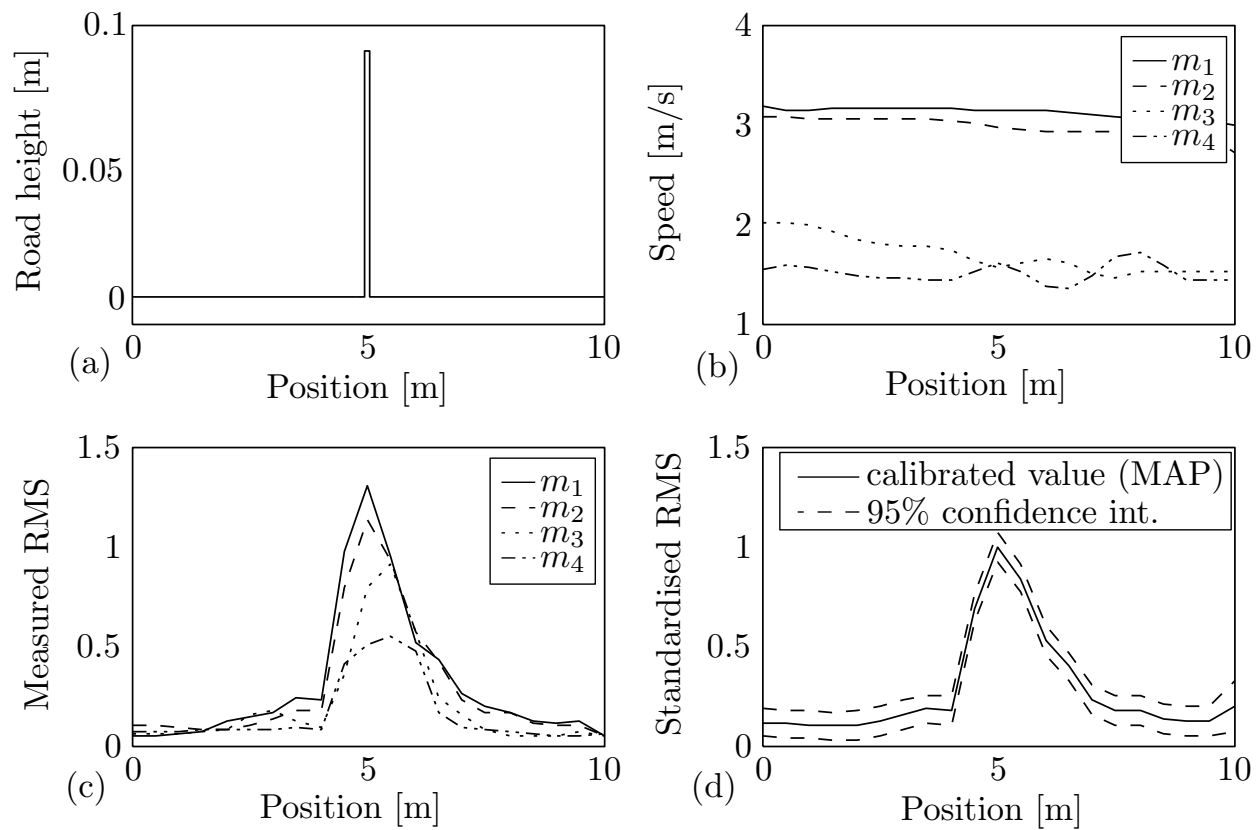

Figure 7: Impulse excitation profile simulated with concrete blocks with severity metric standardised to vehicle speed of $3 \mathrm{~m} / \mathrm{s}$ (four measurements $m_{1}$ to $m_{4}$ )

The response of the vehicle was subsequently measured over the low wavelength ditch which contained two significant humps. The measured road profile is depicted in figure 8 (a). The long wavelength character of most of the profile did not excite the vehicle. The speed dependence of the severity metric over the two humps is clearly illustrated in figures 8 (b) and (c) by the dotted line $\left(m_{2}\right)$ which shows how the driver decelerated for the first hump, but traversed the second hump at fairly high speed. As expected the measured severity metric $\left(m_{2}\right)$ was much smaller for the first hump compared to the second hump. The severity metric which has been standardised to a vehicle speed of $3 \mathrm{~m} / \mathrm{s}$ indicates that both humps are of similar severity. 

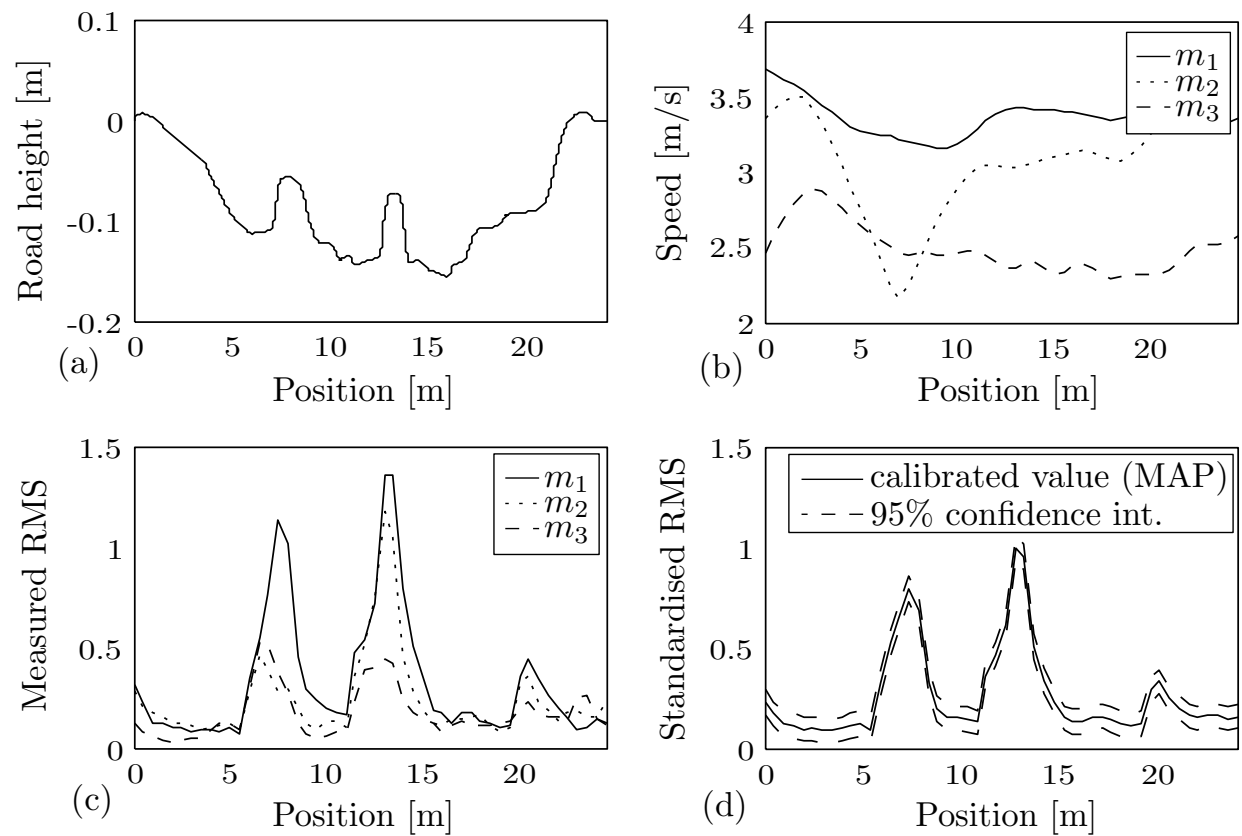

Figure 8: Double hump road profile with RMS metric standardised to vehicle speed of $3 \mathrm{~m} / \mathrm{s}$.

Underground measurements over two $730 \mathrm{~m}$ long road profiles were conducted. The runs from the one road profile were used to generate the parameter priors. Figure 9 indicates the RMS values for the second road profile as estimated at a consistent speed of $3 \mathrm{~m} / \mathrm{s}$. A number of small peaks which are indicative of relatively poor sections of road are identified. The maximum standardised RMS value is 0.4 , which is less than half of the severity of the concrete blocks. 

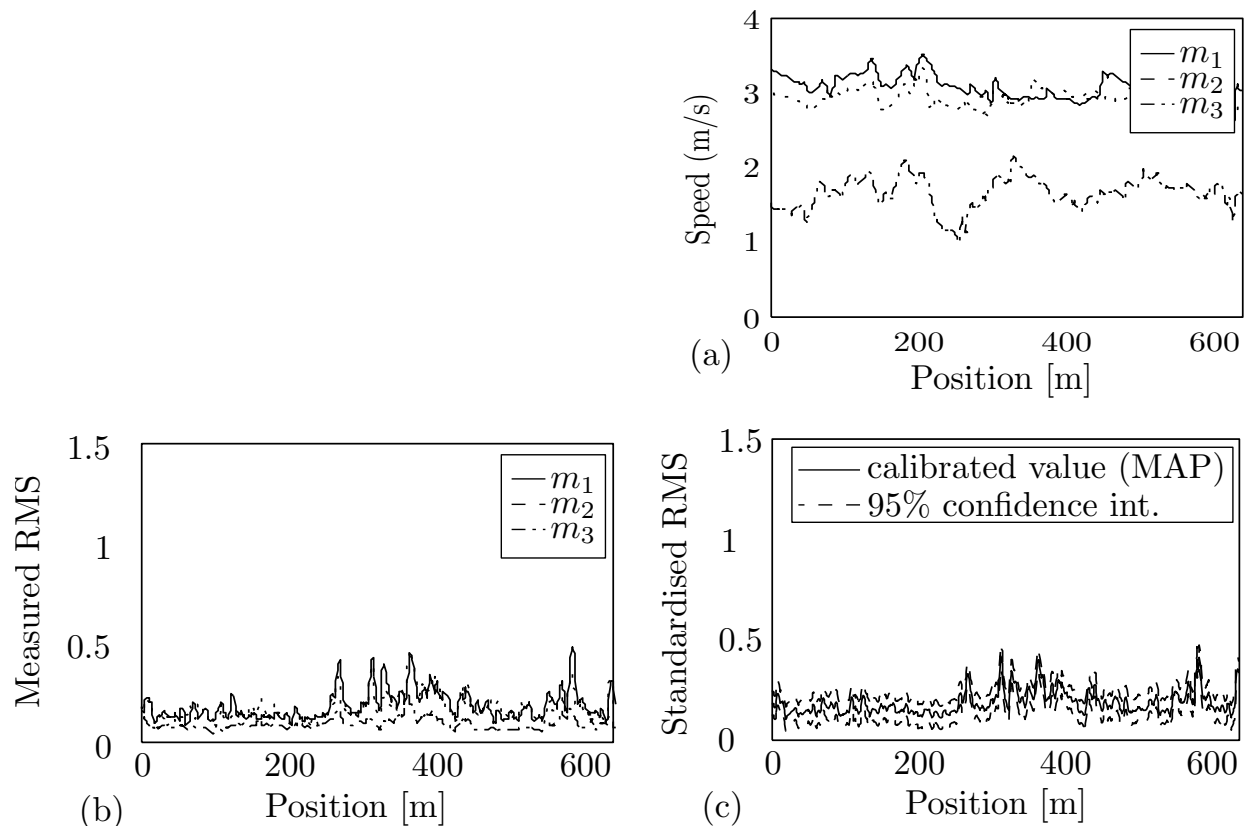

Figure 9: Evaluation of underground road

The correlation between the regression function parameters and the condition of different road surfaces is briefly explored in figures 10 (a) and (b). The estimated RMS values at $3 \mathrm{~m} / \mathrm{s}$ (for the underground measurement) is presented on the x-axis of the figures. A linear speed dependence is assumed, so that each road interval has two estimated parameter values including the offset parameter, and the slope parameter. Only the slope parameter is considered here. The y-axis indicates the value of the slope parameter (dependence on speed). The LSE slope estimates are presented in 10 (a), while the Bayesian (MAP) estimates are shown in 10 (b).
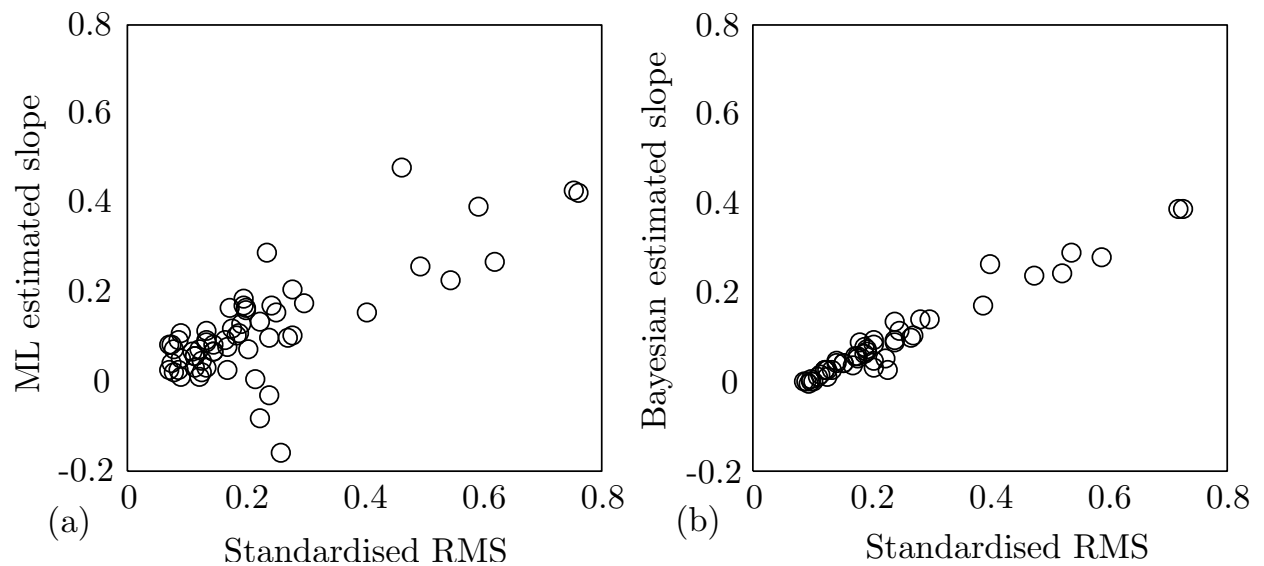

Figure 10: (a) Maximum likelihood parameter (slope) estimates compared to (b) Bayesian paramter (slope) estimates

It is observed that the slopes of the interpolation functions generally increase for more severe roads. Figure 10 (b) illustrate how the inclusion of the prior bias the results closer to the general trend. A number of the LSE slope estimates are negative. This indicates that those LSE calibration functions would predict lower RMS values at 
higher speeds. Although this is not theoretically impossible, it does seem highly unlikely. The prior renders the Bayesian estimates more robust, thereby avoiding negative slope estimates.

\section{Conclusion}

A methodology was proposed which is concerned with the cost-effective monitoring of the condition of haul roads. The methodology is based on estimating a measured vehicle RTRRMS as a function of the vehicle operating conditions. Unique regression functions are estimated for individual road intervals, so as to reflect the spectral properties of that interval. The regression functions are subsequently evaluated at a standardised vehicle operating condition so as to allow for a consistent comparison of the condition of different road intervals.

The regression functions are estimated by means of Bayesian parameter estimation. This allows for the incorporation of a strong prior over the range of expected parameter values. The prior reflects the experience which was gained from road intervals where good quality data were available. The prior subsequently renders it possible to obtain more robust parameter estimates at road intervals where the data quality were constrained.

The methodology was investigated on experimental data which was obtained by measuring the response from a utility vehicle in a coal mine environment. The RMS acceleration response as measured on the vehicle's rear axle was used as RTRRMS metric. The Bayesian framework rendered it possible to estimate a standardised RMS value for the different sections of the road in robust manner, even if only a limited number of measurements were available. While the current study focussed on normalizing the vehicle response with respect to speed, it is anticipated that the same methodology may be implemented to also account for other possibly significant influences on the RTRRMS, such as vehicle acceleration and payload.

It is believed that the proposed methodology may be implemented as a generic haul road monitoring strategy, which could potentially be implemented on a novel fleet of representative vehicles cost effective manner, as it could automatically learn its own priors and regression functions.

[1] R.J. Thompson, A.T. Visser, R.E. Miller, N.T. Lowe, The development of a real-time mine road maintenance management system using haul truck and road vibration signature analysis, The Journal of The South African Institute of Mining and Metallurgy, (June 2003), 265-272.

[2] S. Burningham, N. Stankevich, Why road maintenance is important and how to get it done, The World Bank, Washington DC, TRN-4, (June 2005)

[3] D. Hugo, P.S. Heyns, R.J. Thompson, A.T. Visser, Haul road defect identification using measured truck response, Journal of Terramechanics, 45, (2008) 79-88

[4] H.M. Ngwangwa, P.S. Heyns, F.J.J. Labuschagne, G.K. Kululanga, Haul road defect identification using measured truck response, Journal of Terramechanics, 47, (2010), 97-111

[5] Mechanical vibration and shock - road surace profiles - reporting of measured data, ISO 8606, (E), The International Organization for Standardization, (1995)

[6] M.W. Sayers, T.D. Gillespie, C.A.V. Queiroz, The international road roughness experiment, The World Bank Technical Paper, 45, (1986)

[7] B.E. Quinn, C.C. Wilson, Can dynamic tire forces be used as a criterion of pavement condition, Technical Paper, Joint Highway Research Project, Indiana Department of Transportation and Purdue University, West Lafayette, Indiana, (1963) 
[8] Mechanical vibration and shock - road surace profiles - reporting of measured data, ISO 2631-1, 2nd ed., The International Organization for Standardization, (1997)

[9] A.T.Papagiannakis, J.H.F.Woodrooffe, Suitability of alternative pavement roughness statistics to describe dynamic axle loads of heavy vehicles, Proceedings: the second International Symposium on Heavy Vehicle Weights and Dimensions, Kelowna, British Columbia, (1989)

[10] C.E. Rasmussen, C.K.I. Williams, Gaussian Processes for machine learning, (2006), MIT Press

[11] C.M. Bishop, SpringerLink (Online service), Pattern recognition and machine learning, (2006), Springer New York 